\title{
Distribution of urinary hippuric acid concentrations by ALDH2 genotype
}

\author{
T Kawamoto, K Murata, M Koga, Y Hattori, Y Kodama
}

\begin{abstract}
Objectives-To clarify the relation between the genetic polymorphism of ALDH2 (low $\mathrm{Km}$ aldehyde dehydrogenase) and toluene metabolism.

Methods-The study subjects were 253 toluene workers (192 men and 61 women with an age range of 18-66). The genotypes of ALDH2 were classified by artificial restriction fragment length polymorphism into the homozygous genotype of normal ALDH2 (NN), the homozygous genotype of an inactive ALDH2 (DD), and the heterozygous genotype of normal and inactive ALDH2 (ND). The concentrations of hippuric acid (HA), the main metabolite of toluene, was determined in urine specimens of 253 toluene workers. The HA measurements in previous occupational health examinations were also referenced. The HA concentrations corrected for creatinine (HA/C) were compared with the biological exposure index (BEI) for toluene, which is $2.5 \mathrm{~g} / \mathrm{g}$ creatinine. To estimate the toluene exposures, urinary $o$-cresol concentrations were also determined and compared with another BEI for toluene-that is, $1.0 \mathrm{mg}$ urinary $o$-cresol/g creatinine.
\end{abstract}

Results-Incidence of each genotype in the toluene workers was almost the same as that in non-exposed controls who lived in the same area as the toluene workers. The incidence of each of the three genotypes also did not differ by smoking habit. Mean urinary $H A$ concentrations were not significantly different in the groups with the different genotypes of ALDH2. The HA concentrations of $>70 \%$ of the 890 total samples were $<1.0 \mathrm{~g} / 1$. The number of urine samples $>3.0 \mathrm{~g} / 1$ was 28 $(5 \cdot 4 \%)$ in the NN group and $19(6 \cdot 4 \%)$ in the ND group. No urine samples in the DD group were $>3.0$ g/ HA. The distribution of urinary HA in the DD group was significantly different from those in both the NN and ND groups $(P<0.05)$. Seven $(4.9 \%)$ of the 136 total specimens in the NN group and four (4.7\%) of the 82 total specimens in the ND group exceeded the BEI. There were, however, no urine specimens that exceeded the BEI in the DD group. The maximum HA concentration after correction for creatinine in the DD group was $1.86 \mathrm{~g} / \mathrm{g}$ creatinine. The percentages of urine specimens in which o-cresol concentrations exceeded this
BEI were $14 \cdot 3 \%$ in the NN group, $9 \cdot 1 \%$ in the ND group, and $15.4 \%$ in the DD group. Therefore, the exposure rate for all three genotypic groups of workers was almost the same.

Conclusions-The HA concentrations of toluene workers with ALDH2 DD genotype were lower than those of the NN and ND genotypes when they were exposed to relatively high concentrations of toluene. The exposures of the DD group were suspected to be underestimates because they were based on the BEI for the NN genotype.

(Occup Environ Med 1994;51:817-821)

Keywords: ALDH2 genotype, hippuric acid, toluene

Hippuric acid (HA) is the main metabolite of toluene. Determination of the urinary $\mathrm{HA}$ concentration has been used for biological monitoring of workers exposed to toluene. ${ }^{1}$ Especially in Japan, the measurement of urinary HA has become one of the essential clinical chemistry tests in the occupational health examination for toluene workers since 1989 . About $80 \%$ of the absorbed toluene is first converted to benzyl alcohol by the microsomal mixed function oxidase system, then oxidised to benzoic acid by the alcohol dehydrogenase and aldehyde dehydrogenase system, and finally conjugated to glycine to form HA. ${ }^{2}$

There are two main isoenzymes in aldehyde dehydrogenase (ALDH, EC: $1 \cdot 2.1 \cdot 3)$ namely, ALDH1 (high $\mathrm{Km} \mathrm{ALDH}$ ) and ALDH2 (low Km ALDH). Genetic polymorphism in ALDH2 activity is seen in Japanese and other Mongoloid people, as well as the American Indians. About half of the Japanese people lack ALDH2 activity and experience facial flushing, tachycardia, nausea, and hypotension owing to low acetaldehyde metabolism after drinking alcohol. ${ }^{3}$ Recently, the sequences of $\mathrm{CDNA}$ and genomic DNA of ALDH2 have been clarified. ${ }^{4}$ The catalytic deficiency of aldehyde metabolism is caused by a structural point mutation at amino acid position 487 of the polypeptide subunit coded for by the ALDH2 gene. At this position, a change of Glu to Lys results from the transition of $G(C)$ to $A(T)$ in the DNA. ${ }^{5}$ Thus, it is hypothesised that toluene metabolism in workers deficient in ALDH2 is less than that of workers with normal ALDH2.

With the ALDH2 genotypes, Kawamoto 
et al studied the relation between personal toluene exposure and urinary HA concentrations. ${ }^{6}$ When personal exposure was regressed against urinary HA, the urinary HA concentrations of the workers with a homozygous genotype who were ALDH2 inactive (DD group) were significantly lower than the HA concentrations of the homozygous genotype workers who were ALDH2 normal (NN group) and of the heterozygous genotype workers (ND group). The number of subjects in that study was only 45 , and only six workers belonged to the DD group. To confirm these results, our work has examined the distribution of urinary HA concentrations in a larger number of toluene workers divided according to their ALDH2 genotype.

\section{Subjects and methods}

STUDY SUBJECTS

The study subjects were 253 workers (192 men and 61 women) who handled toluene in electrical parts companies, furniture factories, painting workplaces, and printing companies. The workers were engaged in the cleaning of electrical parts, applying varnish to the surface of furniture, spray painting, and photography. The mean (range) age of the men was 41.0 (18-66) and of the women $47 \cdot 2(21-64)$. All the subjects gave their informed consent. Reference was made to two control groups. One group was made up of 209 men and 51 women who lived in the same area as the toluene workers and who worked in a manufacturing factory and a power plant where organic solvents were not used. Their mean (range) age was 43.3 (19-71) in men and 48.7 (23-67) in women. As a further control, 122 male university students (mean (range) age 23.4 (21-34)) were also studied.

\section{DETERMINATION OF ALDH2 GENOTYPE}

Genomic DNA was isolated from peripheral blood samples $(7 \mathrm{ml})$ by a DNA extractor (Applied Biosystems, model-340A). The ALDH 2 genotype was determined by the artificial restriction fragment length polymorphism (aRFLP) method. ${ }^{7}$ Briefly, exon 12 of the ALDH2 gene, which includes the site of the point mutation, was amplified by the polymerase chain reaction (PCR) method with primers, one of which was a mismatched primer. As a result, the oligonucleotide that was amplified with the normal ALDH2 gene as a template had a restriction site for Mbo II. On the other hand, the oligonucleotide that was amplified with the inactive ALDH2 gene as the template did not have a restriction site for Mbo II. After digestion with Mbo II, the genotypes of ALDH2 were identified on $12 \%$ polyacrylamide gel electrophoresis (PAGE) as the homozygous genotype of normal ALDH2 (NN), the homozygous genotype of inactive ALDH2 (DD), and the heterozygous genotype of normal and inactive ALDH2 (ND). Details are shown by Kawamoto et al. ${ }^{6}$

To confirm the reliability of the aRFLP method, ALDH2 genotypes of 42 samples were determined with an alternative method based on the use of dideoxy guanosine triphosphate (ddGTP). At first 135 base pairs, including the region of the point mutation, were amplified by PCR. A second PCR was performed in a mixed solution of $\mathrm{dCTP}$, dTTP, dATP, ddGTP, first PCR product, and the third primer, which was 5-25 bases upstream from the position of the mutation. Because the DD gene has a mutation from $G$ to $A$, the polymerisation does not end at the mutation position. Differences in the lengths of synthesised oligonucleotides that were subjected to denaturing polyacrylamide gel electrophoresis, indicated the ALDH2 genotypes, as previously reported. ${ }^{8}$

ANALYSIS OF URINARY HIPPURIC ACID, CREATININE, AND $o$-CRESOL

Urine specimens were collected at the end of the work shift, usually from 3:00 to $5: 00 \mathrm{pm}$ and were stored at $-20^{\circ} \mathrm{C}$ until urinary $\mathrm{HA}$ and creatinine were measured. Urine specimens were diluted 51-fold with methanol:water $(1: 1)$. The diluted samples were centrifuged at $3000 \mathrm{rpm}$ for five minutes to pellet denatured protein, and $10 \mu \mathrm{l}$ of the supernatant was applied to a high performance liquid chromatography (HPLC) column (Hitachi L6210, L4250, and D-2500) by a Hitachi autosampler (AS-4000), for analysis by the method of Ogata and Taguchi. ${ }^{9}$

Urinary $o$-cresol was determined by diluting $1.0 \mathrm{ml}$ urine with $4.0 \mathrm{ml}$ double distilled $\mathrm{H}_{2} \mathrm{O}$ and boiling at $100^{\circ} \mathrm{C}$ for 60 minutes with $1.0 \mathrm{ml}$ perchloric acid. After extraction with 4 $\mathrm{ml}$ dichloromethane, the organic phase was examined by a gas chromatograph/mass spectrometer.

REFERENCE TO MEASUREMENTS MADE IN PREVIOUS OCCUPATIONAL HEALTH EXAMINATIONS

The HA concentrations measured in previous occupational health examinations of toluene workers were obtained retrospectively. According to the Japanese Regulation on the Prevention of Organic Solvent Poisoning, the HA concentration ( $\mathrm{g} / \mathrm{l})$ must be measured as a part of an occupational health examination, and creatinine concentrations need not be measured. The urinary HA concentrations in this study were measured at the University of Occupational and Environmental Health (UOEH) laboratory and two other laboratories by the method already mentioned.

\section{STATISTICAL ANALYSIS}

Differences in the HA concentrations were examined by Student's $t$ test and analysis of variance (ANOVA) after log transformation of the urinary HA concentrations, which showed a $\log$ normal distribution. The incidence of the genotypes was examined by the $\chi^{2}$ test.

\section{Results}

The reliability of the aRFLP method used in the study was compared with the dideoxy GTP method (table 1). The ALDH2 genotypes determined by the two different methods 
Table 1 Comparison of the two diagnostic methods for ALDH2 polymorphism

\begin{tabular}{lllrl}
\hline & & \multicolumn{3}{l}{$d d G T P$} \\
\cline { 3 - 5 } & Genotype & $N N$ & $N D$ & $D D$ \\
\hline \multirow{3}{*}{ aRFLP } & NN & 20 & 0 & 0 \\
& ND & 0 & 18 & 0 \\
& DD & 0 & 0 & 4 \\
\hline
\end{tabular}

ddGTP = dideoxy guanosine triphosphate method"; aRFLP = artificial restriction fragment length polymorphism. ${ }^{?}$

corresponded completely. Therefore, we concluded that the aRFLP method of Harada was useful for this study. ${ }^{7}$

Table 2 shows the gene frequencies of ALDH2 genes. The frequency of the normal ALDH2 gene $(N)$ was 0.763 and the inactive gene (D) was 0.237 in male toluene workers; in female workers, they were 0.697 and 0.303 ; the difference between the sexes was not significant. The gene frequencies in toluene workers were compared with those in the nonexposed control group and students. The incidence of each genotype in the toluene workers was not significantly different from those in the non-exposed control group and in students.

The relation between the ALDH2 genotype and smoking was also studied. Table 3 shows that the incidence of each genotype in smokers and non-smokers was almost the same, and the ALDH2 polymorphism did not seem to relate to the smoking habit.

Of 890 HA measurements, 608 samples were analysed at a UOEH laboratory and the others were measured at two laboratories not the UOEH laboratory. To study the reliability of the results from the three different laboratories, 20-33 samples were cross checked by the UOEH laboratory and the two other laboratories. Good agreement between the laboratories was found and no systematic errors were noted in any laboratory. Thus, no correction factors between laboratories were used.

Table 4 shows the urinary HA concentrations by sex, smoking, and ALDH2 genotype. The urinary HA concentrations in female workers were significantly higher than in male workers. There was, however, no significant difference by sex after correction for the creatinine content. Urinary HA concentrations did not differ by smoking, either with or without correction for creatinine. The urinary HA concentration in the female DD group was significantly lower than in the ND and NN groups. The HA concentrations in male workers did not differ by ALDH2 genotypes,
Table 3 The relation between ALDH2 genotype ( $n(\%))$ and smoking in workers

\begin{tabular}{lllll}
\hline & $n$ & $N N$ & $N D$ & $D D$ \\
\hline Smoker & 78 & $48(61 \cdot 5)$ & $24(30 \cdot 8)$ & $6(7 \cdot 7)$ \\
Non-smoker & 66 & $36(54 \cdot 6)$ & $26(39 \cdot 4)$ & $4(6 \cdot 1)$ \\
\hline
\end{tabular}

either with or without correction for creatinine.

Table 5 shows the distribution of urinary HA concentration by the ALDH2 genotype. More than $70 \%$ of the total urine samples from male and female workers contained $<1.0$ g/l HA. The number of urine specimens that were $>3.0 \mathrm{~g} / 1 \mathrm{HA}$ was $24(5.7 \%)$ in men in the $\mathrm{NN}$ group and $14(6.6 \%)$ in men in the ND group. There was no urine specimen in which the HA concentration was $3.0 \mathrm{~g} / \mathrm{l}$ in men in the DD group. The distribution of urinary HA in men in the DD group was significantly different from that in men in the NN and ND groups $(P<0.05)$. In the women, all specimens from DD type workers had HA concentrations from $0-1 \cdot 0 \mathrm{~g} / \mathrm{l}$.

Creatinine concentrations were measured in 237 urine specimens. The HA concentrations corrected for creatinine (HA/C) were compared with the biological exposure index (BEI) of toluene, which was $2.5 \mathrm{~g} / \mathrm{g}$ creatinine. ${ }^{10}$ Seven $(4.9 \%)$ of the 136 specimens in the NN group and four (4.7\%) of the 82 specimens in the ND group exceeded the BEI. There were no samples that exceeded the BEI in the DD group. The maximum HA concentration in the DD group was $1.86 \mathrm{~g} / \mathrm{g}$ creatinine. Although the number of workers in the DD group was too small to give a significant difference, $\mathrm{HA} / \mathrm{g}$ creatinine in the $\mathrm{DD}$ group seemed to be lower than that in the $\mathrm{NN}$ and ND groups.

The figure shows the urinary $o$-cresol concentration by ALDH 2 genotype. The urinary $o$-cresol concentrations in workers who belonged to any of the three ALDH2 genotypes were almost the same. The number of specimens $>0.5 \mathrm{mg} o$-cresol $/ \mathrm{g}$ creatinine was $27(25.4 \%)$ in NN, $12(18.2 \%)$ in ND, and three $(23.1 \%)$ in DD groups. The number of specimens $>1.0 \mathrm{mg} o$-cresol $/ \mathrm{g}$ creatinine was $15(14.3 \%)$ in the NN, six $(9 \cdot 1 \%)$ in the ND, and two $(15.4 \%)$ in the DD group. Therefore, the percentage of workers exposed to high (above threshold limit value (TLV)) concentrations of toluene was almost the same in the three genotypes.

Table 2 Gene frequency of $A L D H 2$ in toluene workers

\begin{tabular}{|c|c|c|c|c|c|c|}
\hline & \multirow[b]{2}{*}{$n$} & \multicolumn{3}{|c|}{ Genotype (n (\%)) } & \multicolumn{2}{|c|}{ Gene frequency } \\
\hline & & $N N$ & $N D$ & $D D$ & $N$ & $D$ \\
\hline \multicolumn{7}{|l|}{ Men: } \\
\hline Toluene workers & 192 & $115(59 \cdot 9)$ & $63(32 \cdot 8)$ & $14(7 \cdot 3)$ & 0.763 & 0.237 \\
\hline Non-exposed control & 209 & $101(48 \cdot 3)$ & $92(44 \cdot 0)$ & $16(7 \cdot 7)$ & 0.703 & 0.297 \\
\hline Students & 122 & $65(53 \cdot 3)$ & $52(42 \cdot 6)$ & $5(4 \cdot 1)$ & $0 \cdot 746$ & $0 \cdot 254$ \\
\hline \multicolumn{7}{|l|}{ Women: } \\
\hline Toluene workers & 61 & $30(49 \cdot 2)$ & $25(41 \cdot 0)$ & $6(9 \cdot 8)$ & 0.697 & 0.303 \\
\hline Non-exposed control & 51 & $29(56.9)$ & $14(27 \cdot 5)$ & $8(15 \cdot 7)$ & 0.706 & $0 \cdot 294$ \\
\hline
\end{tabular}


Table 4 Urinary HA concentration by sex, smoking, and genotype

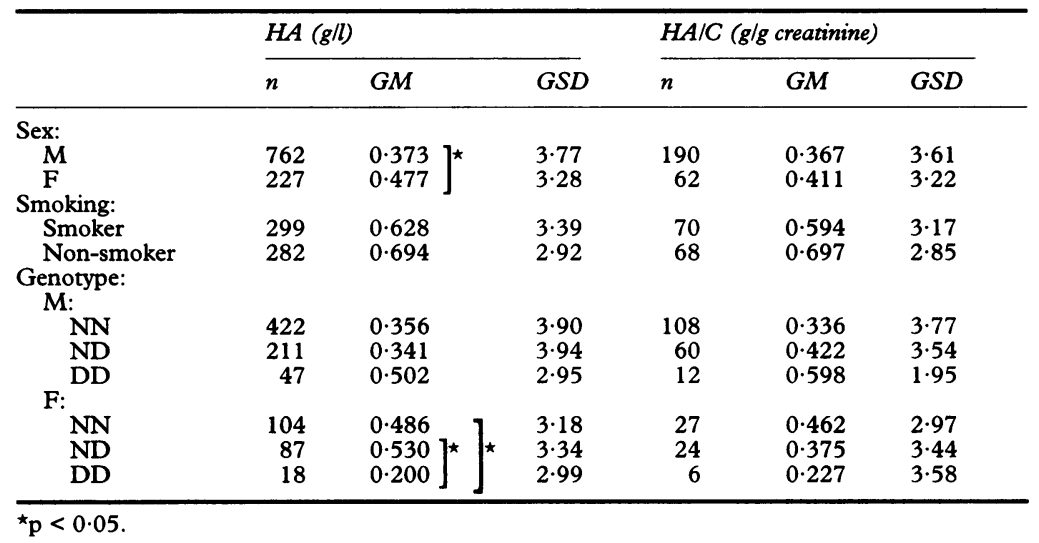

Table 5 Distribution of urinary $H A$ by $A L D H 2$ genotype ( $(\%)$ )

\begin{tabular}{|c|c|c|c|c|c|c|}
\hline \multirow[b]{2}{*}{ Genotype } & \multicolumn{5}{|c|}{ Urinary $H A(g / l)$} & \multirow[b]{2}{*}{ Total } \\
\hline & $0-1$ & $1-2$ & $2-3$ & $3-4$ & $>4$ & \\
\hline \multicolumn{7}{|l|}{ M: } \\
\hline NN & $313(74 \cdot 2)$ & $66(15 \cdot 6)$ & $19(4 \cdot 5)$ & $10(2 \cdot 4)$ & $14(3 \cdot 3)$ & $422(100 \cdot 0)$ \\
\hline $\mathrm{ND}$ & $168(79 \cdot 3)$ & $16(7 \cdot 6)$ & $14(6 \cdot 6)$ & $10(4 \cdot 7)$ & $4(1.9)$ & $212(100 \cdot 0)$ \\
\hline DD & $32(68 \cdot 1)$ & $9(19 \cdot 2)$ & $6(12 \cdot 8)$ & $0(0.0)$ & $0(0.0)$ & $47(100.0)$ \\
\hline Total & $513(75 \cdot 3)$ & $91(13 \cdot 4)$ & $39(5 \cdot 7)$ & $20(2.9)$ & $18(2 \cdot 6)$ & $681(100 \cdot 0)$ \\
\hline \multicolumn{7}{|l|}{ F: } \\
\hline NN & $70(67 \cdot 3)$ & $23(22 \cdot 1)$ & $7(6 \cdot 7)$ & $4(3 \cdot 9)$ & $0(0 \cdot 0)$ & $104(100 \cdot 0)$ \\
\hline ND & $61(70 \cdot 1)$ & $13(14.9)$ & $8(9 \cdot 2)$ & $3(3.5)$ & $2(2 \cdot 3)$ & $87(100 \cdot 0)$ \\
\hline DD & $18(100 \cdot 0)$ & $0(0 \cdot 0)$ & $0(0 \cdot 0)$ & $0(0 \cdot 0)$ & $0(0 \cdot 0)$ & $18(100 \cdot 0)$ \\
\hline Total & $149(71 \cdot 3)$ & $36(17 \cdot 2)$ & $15(7 \cdot 2)$ & $7(3 \cdot 4)$ & $2(1 \cdot 0)$ & $209(100 \cdot 0)$ \\
\hline
\end{tabular}

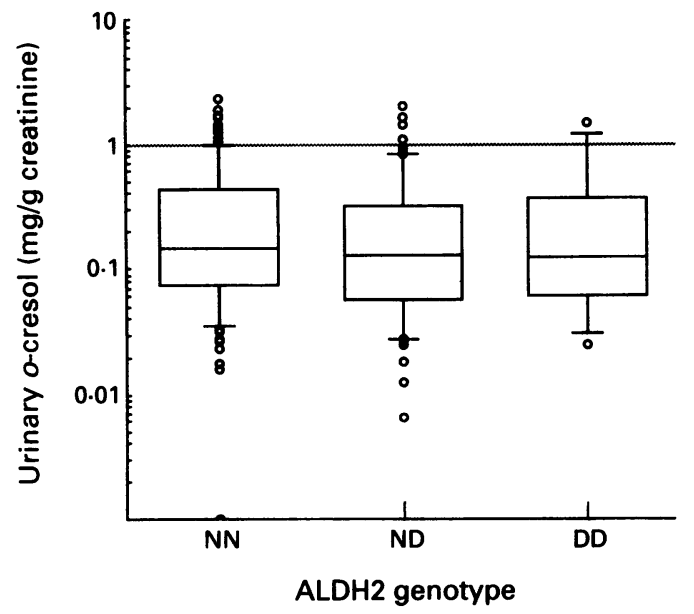

Urinary o-cresol concentration by ALDH2 genotype. Bars indicate 10-90th percentiles, box indicates 25-75th percentiles, and the line in the middle of the box represents the median.

\section{Discussion}

There are several methods to detect point mutations. A method suitable for an epidemiological study must be easy, rapid, reliable, and inexpensive. Genomic DNA samples extracted from a large number of people may not be identical in quantity and quality. The present aRFLP method permitted the determination of ALDH2 genotype from genomic DNA in various conditions, including a low recovery rate of DNA, limited extraction, and impure DNA. The method had to be used to determine the genotype of so many people in such a short period.

Good agreement was obtained in the cross check of measurements of HA concentration between three laboratories. The reasons for the analytical consistency include the following: all three laboratories measured HA by the same method; an official survey for quality control of occupational health examinations is required in Japan ${ }^{11}$; and the three laboratories participated in a voluntary meeting every month to improve the measuring accuracy by the institutes (Society for Improvement of Accuracy and Precision in Occupational Health Examination).

Our study was based on the hypothesis that decreased biotransformation of toluene due to ALDH 2 polymorphism might be responsible for the increased sensitivity to toluene of certain workers: workers with a susceptible genotype ought not to continue to handle toluene: the incidence of such ALDH2 genotypes in toluene workers is predicted to be less than that found in controls. The frequencies of the three ALDH2 genotypes (NN, ND, and DD) in the toluene workers were, however, almost the same as in the non-exposed control group. Thus, it was impossible to conclude whether ALDH2 polymorphism is related to individual sensitivity to toluene. Because the occupational exposure to toluene is not high in Japan (at the most $100-150 \mathrm{ppm}, 370-570 \mathrm{mg} / \mathrm{m}^{3}$ ), it is not expected to affect health. The toluene exposure in glue sniffing, however, may be $>1.0 \%$, and the frequencies of ALDH 2 genes in people addicted to glue sniffing may differ from those in a group of controls.

In the distribution study, $>70 \%$ of the total $\mathrm{HA}$ measurements were $<1.0 \mathrm{~g} / \mathrm{l}$. Kawamoto et al found that urinary HA concentrations ranged from $0 \cdot 02-2 \cdot 13 \mathrm{~g} / 1$ (geometric mean (SD) $0.134(2.52))$ in non-exposed university students. ${ }^{12}$ Urinary HA concentrations were increased not only by toluene exposure but also by the intake of food containing benzoic acid-for example, strawberries, plums, etc. The toluene exposure of most of our study subjects was assumed to be negligible. Subjects in our survey were toluene workers who were required to have a special health examination because of their work with an organic solvent (toluene). The selection criteria for the special health examination included workers who did not handle the solvent but who worked in a room where toluene was used, and also workers who handled toluene only a few times a week or a month, and who did not use toluene on the day of the health examination.

It was suspected that the toluene exposure in the DD group was lower than in the $\mathrm{NN}$ or ND groups, even though we had shown that the $\mathrm{HA}$ and $\mathrm{HA} / \mathrm{g}$ creatinine of urine specimens from the DD group were not $>3.0 \mathrm{~g} / 1$ and $2.0 \mathrm{~g} / \mathrm{g}$ creatinine. Although about $80 \%$ of absorbed toluene is converted to $\mathrm{HA}$ and is excreted in the urine, a small amount of toluene is converted to 0 -cresol. ${ }^{213}$ The metabolism from toluene to $o$-cresol is catalysed by the microsomal mixed function oxidase system, and dehydrogenation by ALDH is not involved in this transformation. Woiwode $e t$ al had previously reported that $o$ cresol in urine was suitable for monitoring exposure to toluene. ${ }^{14}$ To estimate the exposure to toluene, urinary $o$-cresol was measured by gas chromotography-mass spectrometry. 
The American College of Governmental Industrial Hygienists (ACGIH) have proposed $1 \mathrm{mg} / \mathrm{g}$ creatinine of $o$-cresol as a BEI for toluene exposure. ${ }^{19}$ The BEI is defined as the concentration of a determinant that is most likely to be found in specimens collected from healthy workers exposed to chemicals at the TLV (toluene TLV $=100 \mathrm{ppm}$ or 377 $\mathrm{mg} / \mathrm{m}^{3}$ ). To compare the ACGIH BEI of toluene exposure with that of the workers studied here, 232 urine specimens were analysed. About $15 \%$ of the DD group had an estimated exposure $>100 \mathrm{ppm}$ toluene ( 377 $\mathrm{mg} / \mathrm{m}^{3}$ ), the same proportion as in the $\mathrm{NN}$ and ND groups. Therefore, it was concluded that HA concentrations in workers of the ALDH2 genotype DD exposed to toluene were lower than those in workers of the $\mathrm{NN}$ and ND genotypes when exposed to a relatively high concentration of toluene. Furthermore, it is suspected that the workers' exposure in the DD group might have been underestimated.

Based upon these results, a new BEI for each ALDH2 genotype may be necessary. Development of such specific BEIs for various genotypes is especially relevant in Japan because, by law, their urinary HA concentrations must have been determined every six months. Kawamoto et al estimated that BEIs for the NN group should be $2.9 \mathrm{~g} / \mathrm{g}$ creatinine, the ND group should be $3.3 \mathrm{~g} / \mathrm{g}$ creatinine, and the DD group should be $1.9 \mathrm{~g} / \mathrm{g}$ creatinine from the concept that TLVs of all the three genotypes are $100 \mathrm{ppm}$ (377 $\left.\mathrm{mg} / \mathrm{m}^{3}\right) .{ }^{6}$ Before taking such a step, however, some problems remain to be solved. The first is whether the TLV of toluene (now $100 \mathrm{ppm}$, $377 \mathrm{mg} / \mathrm{m}^{3}$ ) is suitable for all the three genotypes. Although we showed that HA formation from toluene in the DD group was lower than that in the NN and ND groups, it is unknown which groups are sensitive to toluene, slow metabolisers, or fast metabolisers. Ideally TLVs of the three ALDH2 genotypes should be determined first from dose-response studies between toluene exposure and subjective and objective symptoms. Then from the regression lines of personal exposures and urinary HA concentrations against the three ALDH2 genotypes that have been reported by Kawamoto et al, ${ }^{6}$ the real BEI of each genotype should be determined by their respective TLVs. Other points to be considered are the ethical justification for the procedure and how to meet the cost of the genotyping.

We thank Dr Chon Shoaf, Hazardous Pollutant Assessment Branch, US EPA, for his useful discussion, and are grateful to Miss Miyuki Shiraishi for her technical assistance. This work was supported in part by a special grant for occupational health studies from the Ministry of Labor of Japan.

1 Ogata $M$, Taguchi T. Quantitative analysis of urinary glycine conjugates by high performance liquid chromatography: excretion of hippuric acid and methylhippuric acids in the urine of subjects exposed to vapours of toluene and xylenes. Int Arch Occup Environ vapours of toluene and
Health 1986;58:121-9.

2 International programme on chemical safety. Environmental health criteria 52, toluene. Geneva: WHO, 1985 . mental hea

3 Harada S. Polymorphism of aldehyde dehydrogenase and its application to alcoholism. Electrophoresis 1989;10: 652-5.

4 Hsu LC, Bendel RE, Yoshida A. Genomic structure of human mitochondrial aldehyde dehydrogenase gene. Genomics 1988;2:57-65.

5 Crabb DW, Edenberg HJ, Bosron WF, Li TK. Genotypes for aldehyde dehydrogenase deficiency and alcohol sensitivity. $\mathcal{f}$ Clin Invest 1989;83:314-6.

6 Kawamoto T, Matsuno K, Kodama Y, Murata K, Matsuda S. ALDH2 polymorphism and biological monMatsuda S. ALDH2 polymorphism and biological mon-

7 Harada S. New strategy for detection of ALDH2 mutant. Alcohol and Alcoholism 1993;(suppl 1A):11-3.

8 Kawamoto T, Matsuno M, Arashidani K, Yoshikawa M, Oyama T, Hattori Y, Kodama Y. Biological monitoring of toluene and DNA polymorphism in humans. Int Arch Occup Environ Health 1993;65:S131-3.

9 Ogata M, Taguchi T. Simultaneous determination of urinary creatinine and metabolites of toluene, xylene, styrene, ethylbenzene and phenol by automated high performance liquid chromatography. Int Arch Occup Environ Health 1988;61:131-40.

10 American Conference of Governmental Industrial Hygienists. Threshold limit values for chemical substances and physical agents and biological exposure indices. and physical agents and biological

11 Sugita M, Harada A, Taniguchi M, Saito M, Imaizumi K, Kitamura M, et al. Quality control program on biological monitoring by Japan Federation of Occupational Health Organizations. Int Arch Occup Environ Health 1991;62: 569-77.

12 Kawamoto T, Matsuno K, Arashidani K, Yoshikawa M, Oyama T, Hattori Y, Kodama Y. Urinary concentration of hippuric acid, phenol and cresols. Proceedings of the 34th annual meeting of the fapan Society of Air Pollution. Chiba City: Japan Society of Air Pollution, 1993:551.

13 Antti-Poika M, Kalliokoski P, Haenninen O. Toluene. In: Snyder R, ed. Ethyl Browning's toxicity and metabolism of Snyder R, ed. Ethyl Browning's toxicity and metabolism of
industrial solvents. Vol 1 hydrocarbons. Amsterdam:

14 Woiwode W, Wodarz R, Drysch K, Weichardt $H$. Metabolism of toluene in man: gas chromatographic determination of $o_{-}, m$ - and $p$-cresol in urine. Arch Toxicol 1979;43:93-8.

\section{Rejected manuscripts}

From February 1994, authors whose submitted articles are rejected will be advised of the decision and one copy of the article, together with any reviewers' comments, will be returned to them. The fournal will destroy remaining copies of the article but correspondence and reviewers' comments will be kept. 